\title{
Power Quality Evaluation of Distribution Network Containing Microgrid Based on PCNN
}

\author{
Ni Fuyin ${ }^{1,2}$, Li Zhengming ${ }^{1, *}$ and Pan Tianhong ${ }^{1}$ \\ ${ }^{I}$ School of Electrical and Information Engineering, Jiangsu University, Jiangsu Zhengjiang, 212013, P.R. China \\ ${ }^{2}$ School of Electrical and Information Engineering, Jiangsu University of Technology, Jiangsu Changzhou, 213001, P.R. \\ China
}

\begin{abstract}
Aiming at the nonlinear relationship between the evaluation indexes and the complex grade of power quality, a power quality evaluation method is proposed based on model of pulse coupled neural network (PCNN). The operating schematic is analyzed according to PCNN modeling. The input power quality grade is controlled by threshold value, and the evaluation grade is output by neuron ignition. The evaluation methods between PCNN and fuzzy neural network are compared through the measured data, the results indicate that PCNN method is more scientific and accurate, and provides a new method to solve the problem on power quality evaluation of distribution network containing microgrid.
\end{abstract}

Keywords: Distribution network, microgrid, PCNN, power quality, power quality evaluation.

\section{INTRODUCTION}

In recent years, microgrid gets fast development on account of its advantages such as flexible power generation, less investment, low energy loss, friendly environmental protection, etc. However, due to microgrid generation inherent characteristics of intermittent and volatility with the gradual increase of microgrid permeability, the power quality problems like voltage fluctuation and harmonic appear in the distribution network. The power quality problems of distribution network incorporating microgrid are becoming prominent increasingly. So it has great significance to evaluate power quality of distribution network containing microgrid $[1,2]$. It is important to study the scientific and effective method of evaluating the power quality.

The problems of nonlinear relationship exist between the evaluation indexes and the complex grades of power quality. At present, the power quality assessment methods around domestic and foreign are mainly concentrated in the following aspects: the way based on analytic hierarchy process [3], the way based on fuzzy mathematics $[4,5]$, the way based on radar map and neural network [6] and so on, improved or different combinations algorithm as well [3, 7-9]. Evaluation systems have some uncertainty because the way based on statistics, vector algebra and fuzzy mathematics, has the subjectivity in different degrees [10]. From another aspect, the related power quality assessment algorithms in references are primarily aiming at main grid power quality assessment. But microgrid has the feature of intermittence and randomness, the measured data are large and dynamic compared to main

*Address correspondence to this author at the School of Electrical and Information Engineering, Jiangsu University, Jiangsu Zhengjiang, 212013, P.R. China; Tel: 13705286653; E-mail: lzming@ujs.edu.cn power grid power. And it is unsuitable for microgrid to assess power quality by using complex algorithms or large amount of calculations.

A power quality evaluation method based on model of pulse coupled neural network (PCNN) [11] is proposed in order to evaluate the distribution network containing microgrid in real time. The internal action items of PCNN neuron which is modulated by feedback-input and link-input is to be set up. Then input power quality grade is to be controlled by threshold value, the evaluation grade is to be output by neuron ignition, and the microgrid power quality is to be evaluated in real time.

\section{PCNN MODEL ESTABLISHED AND OPERATION MECHANISM ANALYSIS}

PCNN is one of neural network models, and it is related to the explanation of primary visual cortex of cats neural excitation oscillation phenomena which was proposed by Eckhorn [12]. It has arisen as the new neural network both at home and abroad in recent years. PCNN belongs to the single artificial neural network compared with traditional multilayer neural network. It's a kind of self-supervised and selflearning network, which is well applied widely to combined optimization decision, image processing, feature extraction and so on [13].

A single neuron model of PCNN is composed of three sections: receiving section, modulation section and pulse generator section. The main structure is shown in Fig. (1) [14].

Receiving section: It accepts input from other neurons and the external. Once the inputs are received, they are transferred by two channels immediately. Among them, a channel 


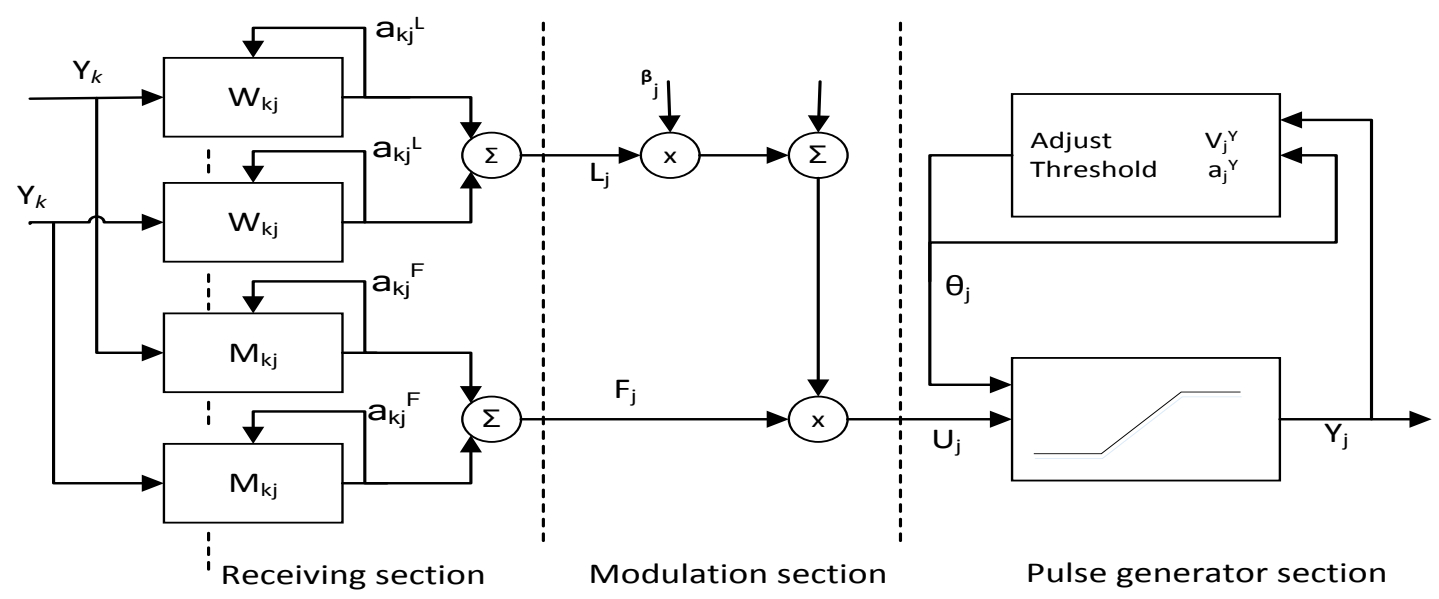

Fig. (1). A single neuron model of PCNN.

is called channel L, as shown in Eq. (1), the other one is called channel F, as shown in Eq. (2).

$$
\begin{aligned}
& L_{j}=\sum_{k} L_{k j}=\sum\left[W_{k j} e\left(-\alpha_{k j}^{L} t\right)\right] \otimes Y_{k}(t)+J_{j} \\
& F_{j}=\sum_{k} F_{k j}=\sum\left[M_{k j} e\left(-\alpha_{k j}^{F} t\right)\right] \otimes Y_{k}(t)+I_{j}
\end{aligned}
$$

Where, $W_{\mathrm{kj}}$ and $M_{\mathrm{kj}}$ are the weights of synaptic junction; $\alpha_{k j}^{L}$ and $\alpha_{k j}^{F}$ are the time constants; $J_{j}$ and $I_{j}$ are the input constants; $Y_{k}(t)$ is the information whether the neuron is firing or not.

Modulation section: Firstly, $L_{i}$ which comes from channel $\mathrm{L}$ adds a positive offset, then the summation multiplies $F_{i}$ which comes from channel $\mathrm{F}$ to modulate, just as shown in Eq. (3), the offset of the model is attributed to be 1.

$U_{j}=F_{j}\left(1+\beta_{j} L_{j}\right)$

Where, $U_{j}$ is defined as the modulation signal by multiplication; $\beta_{j}$ is defined as the linking intensity.

Pulse generator section: It is composed of comparator whose threshold is variable, and pulse generator. When the pulse generator is open, the generating pulse frequency is constant. Neuron threshold gets rapid improvement through feedback as soon as neuron outputs a pulse sequence. When the neuron threshold $\theta_{i}$ is more than $U_{i}$, pulse generator is turned off and stop generating pulse. Then the threshold is decreased exponentially. The pulse generator is open, the neuron is ignited in the same time and outputs a pulse or pulse sequence after the threshold is lower than $U_{j}$. Hence, the maximum frequency of pulse output by neuron is no more than frequency of pulse output by pulse neuron. The expression of pulse generator section is shown in Eq. (4) and Eq. (5):

$$
\begin{aligned}
& \frac{d \theta}{d t}=-\alpha_{j}^{T} \theta_{j}+V_{j}^{T} Y(t) \\
& Y_{j}=\operatorname{Step}\left(\mathrm{U}_{j}-\theta_{j}\right)
\end{aligned}
$$

Where, $V_{j}^{T}$ is the range coefficient of threshold, and $V_{j}^{T}$ is the time constant of threshold; $Y$ is pulse output. One pulse is output if neuron is ignited every time. Then the comparator and pulse generator of the pulse generator section can be substituted for $Y$ output by a step function.

The characteristics of PCNN include variable threshold and periodic pulse generation according to its structure. The neuron outputs pulse once the internal action item is more than dynamic threshold. Then dynamic threshold decays exponentially when it rises to maximum immediately. The neuron generates pulse again as soon as threshold value decreases to be less than internal action items. The neuron which outputs pulse periodically and the adjacent neuron whose feature is similar, get mutual acquisition and generates pulse in synchronization through non-linear modulation. The ignition state of spatial pulse has been observed in different times which reveals the phenomena of dynamic autowave transmission.

To use PCNN method for decision evaluation is inspired by character of auto-wave transmission. The linking input section of PCNN is equal to the pulse generated previously, which reflects the relation between pre- and post-neuron. Then power quality level is determined by dynamic threshold. And it is easy to classify samples by adjusting threshold value. The training of traditional BP model weight is omitted and the complexity is decreased. So the method of PCNN is especially suitable for power quality evaluation on microgrid whose power is unstable. 


\section{POWER QUALITY EVALUATION METHOD BASED ON PCNN MODEL}

Voltage dip, voltage deviation, harmonic, frequency deviation, three-phase unbalance, fluctuation and flicker, reliability, and service indicators are taken as evaluation indexes according to the requirements of internationally recognized definition. These power quality indexes help compose factor sets of evaluation object. Then they are divided into five grades and defined as excellent, good, medium, qualified and unqualified respectively.

To evaluate power quality of distribution network based on PCNN model, the samples are generated uniformly and randomly in the scope of the value of power quality grade. They are input in the form of pulse afterwards. The threshold is adjusted by pulse output, the power quality level is determined through dynamic threshold value. The specific steps are as follows:

Step1: The number scale of the power quality grade and single index classification level is normalized. Suppose the power quality grade and single index classification level is

$\{[a(i, j), b(i, j)], i=1,2 \mathrm{~L} 5, j=1,2 \mathrm{~L} 9\}$,

where, $a(i, j), b(i, j)$ denotes the upper limit value and lower limit value of the variation interval in the power quality grade $i$ and the evaluation index $j$ respectively. The term $i$ is the power quality grade, and term $j$ is the evaluation grade. In order to eliminate the dimension of upper and lower limit and unify the variation range of limitation value, the following Eq. (6) is used to normalize value and set to inter$\operatorname{val}(0,1)$

$$
\begin{aligned}
& a^{*}(i, j)=\left[a(i, j)-a_{\text {min }}(j)\right] /\left[a_{\text {max }}(j)-a_{\text {min }}(j)\right] \\
& b^{*}(i, j)=\left[b(i, j)-b_{\text {min }}(j)\right] /\left[b_{\text {max }}(j)-b_{\text {min }}(j)\right]
\end{aligned}
$$

Step 2: To produce uniform random number from variation interval $\left[a^{*}(i, j), b^{\cdot}(i, j)\right]$. The 100 indexes samples $x(k, j)$ are generated, and corresponding value of standard grade $y(k)$ is $i$. Therefore, the standard sample set of power quality evaluation $\{x(i, j), y(k), k=1,2 \mathrm{~L} n k, j=1,2 \mathrm{~L} 9\}$ is got, where $n k$ is the quantity of samples.

Step 3: Suppose linking weight and threshold value of initial linking section to be $0, V_{L}$ is linking range coefficient, and its value is $1 . Y_{k l}(n-1)$ is defined as information that neuron $x(k, j)$ ignites. Channel $\mathrm{F}$ is used to calculate Feedback input and linking input, as shown in Eq. (7) and Eq. (8):

Feedback input: $F_{i j}(n)=x(k, j)$

linking input: $L_{i j}(n)=V_{L} \sum_{k, l} W_{i j, k l} Y_{k l}(n-1)$
Step 4: Modulation. The internal action item of neuron $x(k, j)$ which is generated by modulation of feedback input and linking input, is shown as Eq. (9):

$U_{i j}(n)=F_{i j}(n)\left(1+\beta L_{i j}(n)\right)$

Where, the term $\beta$ is the linking weight and its value is 0.01 ; pulse generator of neuron $x(k, j)$ outputs binary value by step function from internal action item. The threshold $\theta_{i j}$ is the auto-adjusted according to the ignition state of neuron $x(k, j)$. If the neuron ignites, and $\theta_{i j}$ is adjusted, it is shown in Eq. (10):

$\theta_{i j}(n)=e^{-\alpha \theta} \theta_{i j}(n-1)+V_{\theta} Y_{i j}(n-1)$

Where, the term $\alpha_{\theta}$ is the time decay constant, $V_{\theta}$ is the threshold constant.

Step 5: Pulses generation. As is shown in Eq. (11):

$Y_{i j}(n)=\left\{\begin{array}{l}1, U_{i j} \geq \theta_{i j} \\ 0, U_{i j}<\theta_{i j}\end{array}\right.$

In this simplified model, threshold $\theta_{\mathrm{kj}}$ is the standard of evaluation index. To evaluate the power quality by running the PCNN model, only consider threshold value; the input grade of model is controlled by threshold. The neuron $x(k, j)$ ignites and outputs power quality evaluation grade as soon as $Y_{i j}(n)$ equals to 1 .

\section{CASE STUDY AND ANALYSIS}

Considering environment under the condition that voltage is $380 \mathrm{~V}$, grade limitation table of the power quality index is shown in Table $\mathbf{1}$.

The grade limitation value of evaluation index is normalized, as is shown in Table 2.

The 10 samples are yielded on average in the range of all grades values by Matlab in Table 2. Then these samples are classified by PCNN model one by one and the corresponding grade is obtained. The rationality of model is checked by standard of Table 2 . The measured data of power quality on different observation point in a certain area is shown in Table 3 [9].

Then the normalized measured data is shown in Table 4.

According to the five observation points in a given area of power quality measured data as shown in Table $\mathbf{3}$, these data are normalized as shown in Table 4. Then the power quality evaluation is done based on PCNN model; the evaluation result is shown in Table 5. 
Table 1. Grade limitation of power quality evaluation index.

\begin{tabular}{|c|c|c|c|c|c|}
\hline \multirow[t]{2}{*}{ Evaluation index } & \multicolumn{5}{|c|}{ grade limit } \\
\hline & Q1 & Q2 & Q3 & Q4 & Q5 \\
\hline Voltage deviation & $c=1.20$ & $\approx=3.00$ & $c=4.50$ & $\approx=7.00$ & $>=7.00$ \\
\hline Transient vol tage drop & $>=0.90$ & $>=0.80$ & $>=0.50$ & $>=0.10$ & $c=0.10$ \\
\hline Three phase unbalance & $c=0.50$ & $c=1.00$ & $c=1.50$ & $c=2.00$ & $>=2.00$ \\
\hline Voltage fluctuation & $c=0.50$ & $c=1.00$ & $c=1.50$ & $<=2.00$ & $>=2.00$ \\
\hline Voltage flicker & $c=0.20$ & $\approx=0.50$ & $c=0.80$ & $c=1.00$ & $>=1.00$ \\
\hline Voltage harmonic & $c=1.00$ & $c=2.00$ & $c=3.00$ & $c=5.00$ & $>=5.00$ \\
\hline Frequency deviation & $c=0.05$ & $c=0.10$ & $s=0.150$ & $c=0.20$ & $>=0.20$ \\
\hline The power supply reliability index & $>=0.95$ & $>=0.85$ & $>=0.80$ & $>=0.70$ & $c=0.70$ \\
\hline Service index & $>=0.90$ & $>=0.80$ & $>=0.70$ & $>=0.60$ & $c=0.60$ \\
\hline
\end{tabular}

Table 2. Grade limitation normalization of power quality evaluation index.

\begin{tabular}{|c|c|c|c|c|c|}
\hline \multirow{2}{*}{ Evaluation index } & \multicolumn{4}{|c|}{ Grade limit } & \multirow[b]{2}{*}{ Q5 } \\
\hline & Q1 & Q2 & Q3 & Q4 & \\
\hline Voltage deviation & $\varepsilon=1.70$ & $<=0.43$ & $\varepsilon=0.64$ & $\varepsilon=1.00$ & $>=1.00$ \\
\hline Transient vol tage drop & $>=1.00$ & $>=0.89$ & $>=0.55$ & $>=0.11$ & $\varepsilon=0.11$ \\
\hline Three phase unbalance & $c=0.25$ & $c=0.50$ & $c=0.75$ & $c=1.00$ & $>=1.00$ \\
\hline Voltage fluctuation & $\varepsilon=0.25$ & $\varepsilon=0.50$ & $\varepsilon=0.75$ & $c=1.00$ & $>=1.00$ \\
\hline Voltage flicker & $c=0.20$ & $c=0.50$ & $c=0.80$ & $c=1.00$ & $>=1.00$ \\
\hline Voltage harmonic & $\varepsilon=0.20$ & $c=0.50$ & $\varepsilon=0.80$ & $c=1.00$ & $>=1.00$ \\
\hline Frequency deviation & $c=0.25$ & $\varepsilon=0.50$ & $c=0.75$ & $c=1.00$ & $>=1.00$ \\
\hline The power supply reliability index & $>=1.00$ & $>=0.89$ & $>=0.84$ & $>=0.74$ & $\approx=0.74$ \\
\hline Service index & $>=1.00$ & $>=0.89$ & $>=0.78$ & $>=0.67$ & $\varepsilon=0.67$ \\
\hline
\end{tabular}

Table 3. The measured data of power quality in a certain area.

\begin{tabular}{|lccccc|}
\hline The measured data & observation 1 & 2 & 3 & 4 & 5 \\
\hline Voltage deviation & 3.212 & 6.68 & 4.35 & 5.33 & 4.22 \\
Transient vol tage drop & 79.63 & 15.89 & 51.56 & 58.56 & 48.63 \\
Three phase unb alance & 0.83 & 1.36 & 1.35 & 1.74 & 1.83 \\
Voltage fluctuation & 1.33 & 1.53 & 1.95 & 1.37 & 1.58 \\
Voltage flicker & 47.3 & 84.7 & 63.4 & 82.6 & 82,8 \\
Voltage harmonic & 1.72 & 4.28 & 2.67 & 3.36 & 4.57 \\
Frequency deviation & 0.0922 & 0.1562 & 0.1180 & 0.1787 & 0.1892 \\
The power supply reliability ind $\mathrm{x}$ & 0.833 & 0.762 & 0.796 & 0.740 & 0.764 \\
Service index & 0.832 & 0.713 & 0.864 & 0.684 & 0.783 \\
\hline
\end{tabular}


Table 4. The grade limit of normalization of the power quality evaluation index.

\begin{tabular}{|lccccc|}
\hline The measured data & observation 1 & 2 & 3 & 4 & 5 \\
\hline Voltage deviation & 0.46 & 0.95 & 0.62 & 0.76 & 0.60 \\
Transient vol tage drop & 0.88 & 0.18 & 0.57 & 0.57 & 0.54 \\
Three phase unbalance & 0.415 & 0.68 & 0.675 & 0.87 & 0.915 \\
Voltage fluctuation & 0.665 & 0.765 & 0.975 & 0.685 & 0.79 \\
Voltage flicker & 0.473 & 0.847 & 0.634 & 0.826 & 0.828 \\
Voltage harmonic & 0.344 & 0.856 & 0.534 & 0.672 & 0.914 \\
Frequency deviation & 0.461 & 0.781 & 0.59 & 0.8935 & 0.946 \\
The power supply reliability ind & 0.877 & 0.762 & 0.802 & 0.779 & 0.804 \\
Service index & 0.924 & 0.792 & 0.96 & 0.76 & 0.87 \\
\hline
\end{tabular}

Table 5. Results comparison of power quality evaluation methods on observation points.

\begin{tabular}{|lccccc|}
\hline The measured data & observation 1 & 2 & 3 & 4 & 5 \\
\hline Voltage deviation & 0.46 & 0.95 & 0.62 & 0.76 & 0.60 \\
Transient voltage drop & 0.88 & 0.18 & 0.57 & 0.52 & 0.54 \\
Three phase unb alance & 0.415 & 0.68 & 0.675 & 0.87 & 0.915 \\
Voltage fluctuation & 0.665 & 0.765 & 0.975 & 0.685 & 0.79 \\
Voltage flicker & 0.473 & 0.847 & 0.634 & 0.826 & 0.828 \\
Voltage harmonic & 0.344 & 0.856 & 0.534 & 0.672 & 0.914 \\
Frequency deviation & 0.461 & 0.781 & 0.59 & 0.8935 & 0.946 \\
The power supply reliability index & 0.877 & 0.762 & 0.802 & 0.779 & 0.804 \\
Service index & 0.924 & 0.792 & 0.96 & 0.76 & 0.87 \\
\hline
\end{tabular}

Comparing the method based on the PCNN with the method based on fuzzy neural network [6], evaluation results are basically close. The difference lies in observation points 1. PCNN method is less one grade compared to fuzzy neural network method on observation point 1 . The voltage sag on observation point 1 is relatively serious, and power quality evaluation grade is more reasonable as the medium based on PCNN.

\section{CONCLUSION}

According to the characteristics that the measured data is very variable and uncertain, PCNN model is established. And it is applied to the study on the decision and evaluation of distribution network on the basis of the analysis of the running mechanism of auto-wave. The different feedback input equation, linking input equation and threshold modulation equation are introduced based on the structure of PCNN single neuron, and the satisfied evaluation is obtained.
The results show that the method is scientifically accurate, and it can achieve evaluation in real time. It can provide a new method to solve the problem on power quality evaluation of distribution network containing microgrid.

\section{CONFLICT OF INTEREST}

The authors confirm that this article content has no conflict of interest.

\section{ACKNOWLEDGEMENTS}

Supported by National Natural Science Foundation of China (51477070).

Supported by Natural Science Foundation of Jiangsu University of Technology (KYY14001).

Supported by A Project Funded by the Priority Academic Program Development of Jiangsu Higher Education Institutions (PAPD). 


\section{REFERENCES}

[1] S.Z. Wang, S. Gao, H. Li, and L. Zhang, "Evaluation of power quality in grid planning scheme with wind power integration", Transaction of China Electro Technical Society, vol. 28, pp. 56-65, 2013.

[2] H.Y. Li, and J.P. Min, "Power quality assessment considering characteristics of distributed generation system", Electric Power Automation Equipment, vol. 34, pp. 31-36, 2014

[3] Z.M. Li, J.H. Zhang, and M.J. Chen, "Fuzzy comprehensive evaluation of enterprise's orderly power utility based on analytic hierarchy process", Power System Protection and Control, vol. 41, pp. 136-141, 2013.

[4] W.X. Xu, and X.M. Liu, "Study on the accuracy of Comprehensive evaluation method based on fuzzy set theory", Journal of Systems Engineering and Electronics, vol. 2, pp. 330-334, 2005.

[5] P.C. Qiao, Z.G. Wu, and H. Li, "Power quality synthetic evaluation based on improved radar chart", Electric Power Automation Equipment, vol. 31, pp. 88-92, 2011.

[6] L. Zhou, Q.L. Li, H.Y. Liu, F. Zhang, and Y.J. Qu, "Evaluation of power quality by fuzzy artificial neural network", High Voltage Engineering, vol. 33, pp. 66-69, 2007.

[7] J.H. Liu, L.F. Luo, Z.W. Zhang, and J.Z. Xu, "A new method for power quality comprehensive evaluation considering the analysis of sequence stability", Proceedings of the CSEE, vol. 33, pp. 70-76, 2013.

[8] Z.X. Jing, and R. Hu, "Power quality evaluation based on twostage method and its sensitivity analysis", Electric Power Automation Equipment, vol. 33, pp. 57-62, 2013.

[9] Z.M. Li, S. Shi, T.H. Pan, and W.H. Chen, "A synthetic power quality assessment based on grey correlation analysis and TOPSIS method", Power System Protection and Control, vol. 42, pp. 14-19, 2014.

[10] Y.Y. Liu, P. Dai, Y.H. Xu, and Y.F. Liu, "Comparison of power quality comprehensive evaluation methods", Electric Power Automation Equipment, vol. 28, pp. 93-97, 100, 2014.

[11] J. L. Johnson, "Padgett M L.PCNN models and applications", IEEE Transactions on Neural Networks, vol. 10, pp. 591-598, 1999.

[12] R. Ekkhorn, H. J. Reithhoeck, M. Arndt, and P. Dicke, "Feature linking via synchronization among distributed assemblies: simulations of result from cat visual cortex", Neural Computation, vol. 2, no. 3, pp. 293-307, 1990.

[13] C.H. Yang, B.H. Wang, J. Fu, and Y.G. Xie, "Pulse-coupled neural network model for evaluation of flood disasters", Journal of Catastrophology, vol. 25, pp. 12-15, 2010

[14] Y.D. Ma, F. Shi, and L. Li, "A new kind of impulse noise filter based on PCNN", IEEE ICNNSP, vol. 1, pp. 152-155, 2003.

This is an open access article licensed under the terms of the Creative Commons Attribution Non-Commercial License (http://creativecommons.org/licenses/bync/4.0/) which permits unrestricted, non-commercial use, distribution and reproduction in any medium, provided the work is properly cited. 